\title{
MedienPädagogik
}

Zeitschrift für Theorie und Praxis der Medienbildung

\section{Postkolonial post-digital}

\section{Forschungsfelder und Anschlussstellen für die Medienpädagogik durch eine postkoloniale Perspektive auf eine Post-Digitalität}

\author{
Nina Grünberger
}

\section{Zusammenfassung}

Aktuell werden Begriffe wie Digitalisierung, Mediatisierung, Medialisierung sowie Digitalität und Post-Digitalität viel diskutiert. Diese Diskurse werden vorrangig aus einer Perspektive des Globalen Nordens geführt. Dabei geht es häufig um die 〈sinnvolle〉 Integration (digitaler) Medien in Bildungskontexte und das transformierende Potential für Bildungsinstitutionen von Elementarpädagogik, über Schule bis Hochschule. Versuche der Begriffsbestimmungen von Digitalisierung, Mediatisierung oder auch Post-/Digitalität sind stark von der geopolitischen und sprachlichen Verortung geprägt. Doch die Kultur der Digitalität und die globale digitale Vernetzung der Welt, legen hinsichtlich bestimmter Fragen eine weltumspannende postkoloniale Perspektive nahe. Der vorliegende Beitrag versucht einen möglichen methodologischen Zugang zu einer weltumspannenden Medienpädagogik zu skizzieren und handelt dies entlang ökologischer Implikationen der Ubiquität digitaler Technologie und eines ökologisch-nachhaltigeren Medienhandelns ab. Dem ist eine Begriffsklärung zu Digitalität und PostDigitalität vorangestellt. Der Beitrag schlägt einen interdisziplinären Zugang vor, der medienpädagogische und medienwissenschaftliche Überlegungen sowie «Bildung für nachhaltige Entwicklung» und Postcolonial Studies einbezieht. Daraus ergeben sich medienpädagogische Anschlussstellen und Forschungsfelder. 


\title{
Media education and global environmental challenges. A postcolonial approach of a wide-ranging term of digitality
}

\begin{abstract}
Talking about digitization, mediatization, medialization or post-/digitality we are limited in a perspective of the Global North, mostly dealing with the implementation of (digital) media in educational institutions. Educators, educational researchers as well as politicians are often requiring more technological features, better technical conditions and the taxonomisation of digital skills for countries in the Global North. But this perspective inhibits us to consider a wide-ranging term of digitality which includes e.g. environmental implications of an ubiquitous use of digital media or taxonomies of digital competences for the Global South. To that extant an postcolonial and media archaeological approach is proposed to think digitality consequently wide and far-reaching. This opens up responsibilities and tasks for media-pedagogy in research, as well as for the development of concepts and educational practices.
\end{abstract}

\section{Hinführung}

Der deutschsprachige Diskurs um Digitalisierung, Mediatisierung oder Medialisierung kreist vorrangig um Fragen der Implementierung digitaler Medien in Lern- und Bildungsprozesse und daraus resultierende Implikationen für Bildungsinstitutionen. Dies hat sich auch in politischen Strategien zur 〈Digitalisierung von Bildung〉 manifestiert (vgl. bspw. KMK 2016; BMBWF 2018). Hierbei hat sich weitgehend der Mediatisierungsbegriff durchgesetzt, der häufig heterogen und als «argumentative[r] Allgemeinplatz» (Bettinger und Assmann 2017) in der Medienpädagogik verwendet wird. Soziologische, kommunikations- oder auch medienwissenschaftliche Diskurse dazu bleiben eher unberücksichtigt (vgl. bspw. die Beiträge in Swertz u. a. 2017; Trültzsch-Wijnen 2017). Differenzierte Begriffsschärfungen versuchen die Digitalisierung, Digitalität oder auch Post-Digitalität entlang ihrer Implikationen für das soziale Zusammenleben zu definieren. 
Da wie dort zeigt die Auseinandersetzung vorrangig eine Perspektive des Globalen Nordens ${ }^{1}$. Nur selten finden sich tatsächlich weltumspannende, den Globalen Süden einschliessende Überlegungen. Dies ist angesichts der globalen digitalen Vernetzung, politischer Bestrebungen des Ausbaus digitaler Infrastruktur sowie 〈digitaler skills) und der weitreichenden sozialen, ökonomischen und ökologischen Implikationen der Ubiquität digitaler Technologie verwunderlich.

Der Begriff der Digitalität (vgl. bspw. Stalder 2019) scheint für eine weltumspannende Perspektive sinnvoll, insofern er «gesellschaftsformende» Praktiken ebenso wie "Artefakte, Institutionen und Lebenswelten» (Stalder 2016, S. 16) fokussiert. Die Digitalität betrifft mit ihren Eigenheiten alle Sozialitäten, unabhängig ob des Globalen Nordens oder Südens. Eng damit verbunden ist der Begriff der Post-Digitalität. Dabei meint das Post- vor der Digitalität nicht ein Herauslösen aus dieser, sondern betont die Unvermeidlichkeit der Präfigurierung der Lebenswelt durch digitale Technologie und Algorithmen. Digitale Technologie ist nicht neu und emergent. Das Post- vor der Digitalität verweist auf die unausweichlichen Konsequenzen der Digitalität auf alle Lebensbereiche (Murray 2020, 448). Der vorliegende Beitrag wird zusätzlich die Unvermeidlichkeit der Digitalität für unseren Lebensraum aus weltumspannender Perspektive, also für den Globalen Süden wie Norden zeigen.

Insofern wird im Folgenden aus kritisch-konstruktiver Perspektive eine thematische Hinführung zu einem postkolonialen Verständnis der Post-/Digitalität skizziert. Dabei wird in erster Linie das Forschungsfeld eröffnet. Dies erfolgt zunächst entlang der Begriffsschärfung von Mediatisierung, Digitalität und Post-Digitaliät. Im Anschluss werden methodologische Zugänge entlang einer postkolonialen Perspektive beschrieben, um

1 In der Literatur ist eine Unterscheidung in Industrieländer bzw. -staaten, westliche Welt oder den Globalen Norden in Abgrenzung zu Entwicklungsländern oder Ländern des Globalen Südens gängig. Unabhängig von der Begriffswahl scheint eine vermeintliche Dichotomisierung nur schwer vermeidbar. In diesem Artikel werden daher die Begriffe Globaler Norden sowie Süden verwendet, da diese im aktuellen Diskurs üblicher erscheinen. Zudem wird dabei die Entwicklungsmetapher (wie im Begriff 'Entwicklungsländer') nicht bedient, die nahelegen würde, dass sich der Globale Süden erst richtig entwickeln müsse, will er zu den Industrieländern gehören. Eine glückliche Wortwahl ist es dennoch nicht. 
schliesslich orientiert an ökologischen, ökonomischen und sozialen Implikationen der ubiquitären Nutzung digitaler Technologie konkreter zu werden. Entlang des Lebenszyklus eines Smart-Mobile-Devices werden die weitreichenden Implikationen eines postkolonialen, weltumspannenden Blicks auf die Post-/Digitalität aufgezeigt. Der Beitrag geht also der Frage nach den weitreichenden Forschungsfeldern und Anschlussstellen für die Medienpädagogik nach, die sich durch eine weltumspannende, postkoloniale Perspektive auf die Post-/Digitalität ergeben ${ }^{2}$. Schon früh zeigt sich, dass ein solches Vorhaben ein interdisziplinäres Setting mit Ansätzen aus der Medienwissenschaft und Medienpädagogik, der «Bildung für nachhaltige Entwicklung», des «Globalen Lernens» und der Postcolonial Studies bedarf.

\section{Mediatisierung, Digitalität und Post-Digitalität}

Wie erwähnt, wird im Diskurs der deutschsprachigen Medienpädagogik häufig der Mediatisierungsbegriff bemüht. Zunehmend finden sich auch die Begriffe Digitalität und Post-Digitalität. Diese drei Begriffe werden im Folgenden im Kontext von Bildung und Lernen skizziert.

Mit dem Mediatisierungsbegriff wird meist eine grundlegende Veränderung der Lebenswelt im Sinne eines Mehr an und einer Ubiquität von Medien angesprochen. Damit sind sozio-kulturelle Transformationen verbunden, die auch Bedingungen von Lernen und Bildung betreffen. Mediale Entwicklungen bedingen Lernen und Bildung in ihrer Grundkonstitution. Je nach Wahl einer Leittheorie aus soziologischer (vgl. u. a. Lyotard 1979; Bauman 2000), philosophischer oder anthropologischer (vgl. u. a. Faßler 2009; 2011; 2014) Perspektive, stellen sich die Beschreibungen der Veränderungen von Lernen und Bildung unterschiedlich dar. Dass mediale Entwicklungen und insbesondere die quantitative Zunahme digitaler Medien in der Lebenswelt zu sozio-kulturellen Transformationserscheinungen führen, ist wohl gemeinsamer Nenner vieler Mediatisierungskonzepte. Schon Marshall McLuhan schrieb der Erfindung des Buchsdrucks eine ähnliche fundamentale Veränderung der Gesellschaft zu, indem

2 Die Frage der Verantwortung der Medienpädagogik in diesem Kontext habe ich bereits andernorts ausführlicher diskutiert, daher wird dies an dieser Stelle nur angerissen (Grünberger 2020). 
die «Mechanisierung der Schreibkunst», eine «Zerlegung einer Handfertigkeit in mechanische Glieder» (McLuhan 1962, 172) und damit die Aufgliederung komplexer Arbeitsschritte, die Arbeitsteilung und eine erste Technologisierung von Produktionsprozessen ermöglichte. Nach Hepp $(2014,1)$ ist Mediatisierung «die Wechselbeziehung zwischen medienkommunikativem und soziokulturellem Wandel», die sich quantitativ in einer «zunehmenden zeitliche[n], räumliche[n] und soziale[n] Verbreitung von medienvermittelter Kommunikation» und qualitativ in den Implikationen der medialen Entwicklung für den sozio-kulturellen Wandel zeigt. Mediatisierung ist ein «Metaprozess〉 des Wandels [...] von Kultur und Gesellschaft» (Hepp 2014), der seinen Ausgangspunkt bei vormaligen Massenmedien nahm und nun alle Lebensbereiche entlang einer «Medienlogik», auch ausserhalb der Strukturen von Massenmedien, präfiguriert. Auch die, den Medien «zugrundeliegenden Infrastrukturen» (Hepp 2018b), etwa die Algorithmen-Logik, bedingen soziale Konstruktionen: Algorithmen machen Bestimmtes denk- bzw. sagbar und anders nicht. Der Mediatisierungsbegriff denkt zudem den technologischen, «materialistischen»Kontext mit (Hepp 2018a). Den genannten Ansätzen ist gemein, dass sie nach dem Verhältnis von sozio-kulturellem und medialem Wandel fragen, von einer Prozesshaftigkeit und Historizität ausgehen und nicht an Techniken, sondern am kommunikativen Menschen ansetzten. Doch der Mediatisierungsbegriff ist stets im jeweiligen Kontext zu denken (Hepp 2014). Die «Vielschichtigkeit von Mediatisierung» impliziert jeweils historische, regionale und soziale Spezifika. Doch der aktuelle Mediatisierungsdiskurs wird ausgehend von der Perspektive des Globalen Nordens geführt, obwohl «Mediatisierungsprozesse in unterschiedlichen Regionen der Welt kontextualisiert auf je unterschiedliche Weise auszumachen sind» und es einer «Ent-Westlichung〉 der Mediatisierungsforschung» bedürfe (Hepp 2014).

Der Digitalitätsbegriff geht über den Fokus auf Medien hinaus und thematisiert die digitale Präfigurierung unseres Lebens und unseres Lebensraumes. Nach Stalder, orientiert an McLuhan, ist offensichtlich, «dass alte kulturelle Formen, Institutionen und Gewissheiten erodieren» und «dass sich neue herausbilden, deren Konturen schon recht deutlich zu erkennen sind, nicht mehr nur in Nischen, sondern in der Mitte der Gesellschaft» (Stalder 2019, 9). Die Digitalität zeichnet sich insbesondere durch drei Charakteristika aus: Zum Ersten ist dies die «Referentialität, also die Nutzung 
bestehenden kulturellen Materials für die eigene Produktion», für die das «Auswählen und Zusammenführen» als kulturelle Praktik im Rahmen einer erhöhten Kontingenz zentral ist. Zusätzlich beschreibt die Digitalität neue Formen der «Gemeinschaftlichkeit», die «selbstbezogene Welten» mit eigenen Norm- und Wertvorstellungen hervorbringen und in denen sich «Dynamiken der Netzwerkmacht, [der] Freiwilligkeit und [des] Zwang[s], Autonomie und Fremdbestimmung» in neuer Weise zeigen (Stalder 2019, 9; vgl. dazu auch Faßler 2009). Und schliesslich wird die Kultur der Digitalität durch die «Algorithmizität» charakterisiert, also durch «automatisierte Entscheidungsverfahren, die den Informationsüberfluss reduzieren und formen», so dass daraus Informationen entstehen, die von Menschen verstanden und zur Grundlage ihrer Handlungsentscheidungen gemacht werden können. Die Digitalität zeigt sich in «singulären und kollektiven Handlungen», in «Zeichen und Symbolen», in «Artefakten, Institutionen und Lebenswelten» (Stalder 2019, 16). Dabei denkt Stalder keine Dichotomie zwischen Analog und Digital. Und das Digitale ist nicht abstrakt. Es zeigt sich auch in materieller Hinsicht: oder Materialität und Imaterialität: «[D]ie flüchtigen Impulse digitaler Kommunikation beruhen auf globalen, durch und durch materiellen Infrastrukturen, die von den Minen tief unter der Erfoberfläche, in denen Metalle der Seltenen Erden abgebaut werden, bis ins Weltall, wo Satelliten die Erde umkreisen, reichen. Diese sind in der Alltagserfahrung jedoch kaum sichtbar und werden daher oft ignoriert, ohne dass sie deswegen verschwinden, oder an Bedeutung verlieren.» (Stalder 2019, 18)

Stalders (2019, 18f) Digitalitätsbegriff ist dem Begriff des Post-Digitalen ähnlich. Dabei symbolisiert das Post- vor dem Digitalen insbesondere eine Ablehnung des «ideologischen Ballast[s]», der die Unterscheidung in «alte» und «neue» Medien impliziert. Ebenso soll damit der, dem Digitalen typische Fortschrittsglaube sowie Eigenschaften wie «Immaterialität, Perfektion und Virtualität» verworfen werden (Stalder 2019, 19). Mit der Betonung des Post-Digitalen gerät in den Blick, dass Praktiken und Strukturen, die sich in digitalen Kontexten entwickelt haben, nunmehr auch in anderen Kontexten und Materialitäten zu finden sind. Der Post-Digitalitätsbegriff verweist kritisch darauf, dass sich die Digitalität mit ihren 
Eigenschaften in unterschiedlichste Lebenskontexte, Praktiken, Institutionen und Artefakte eingeschrieben hat und zwar in der Form, dass die Digitalität als solches nicht mehr zwingend wahrgenommen wird (Cramer 2014, 20). Anders formuliert: Das Digitale ist alternativlos geworden und es ist keine Revolution, kein Werden, sondern ein Sein. «Digitaltechnologie [bedeutet] nicht per se Fortschritt und Zukunft», sondern in erster Linie Realität, Lebensmitte (Cramer 2016). Und diese Realität ist gekennzeichnet durch diffuse Relationen und Strukturen:

«With a tendency toward ephemerality, acceleration, confusion about materiality, and multiple layers of signification (infrastructure, interface, content), digital media can seem at odds with a non-field that takes seriously 'what we wear, hear, watch, and eat' [...]. But now more than ever our attention is guided by digitalities: what we wear is responsive to our tracked, mobile bodies; what we hear is intimately personal; what we watch is guided by software keyed to our individual tastes; and, what we eat is served by gig economies and smart crockpots.» (Murray 2020, 442)

Murray vergleicht die Algorithmizität mit der kulturwissenschaftlichen Bedeutung von Jeans als Symbol für Freiheit und Grenzen der Populärkultur: Jeans stehen für Alltagswelt und Partizipation in bestimmten Sub-Gesellschaften.

«Similar to how we still purchase jeans ripped, high-waisted, and wearable in our own image, we invite into our lives the algorithm's banal functionality.» (Murray 2020, 445).

Dass die Digitalität respektive Post-Digitalität auch Lernen und Bildung im Kern bedingt, hat in der Medienpädagogik Eingang gefunden (vgl. bspw. Jörissen und Marotzki 2009; Meder 2004). Doch scheint der Diskurs vorrangig die Perspektive des Globalen Nordens abzubilden und das obwohl die globale digitale Vernetzung und die Idee der Post-/Digitalität einen weltumspannenden Blick geradezu evozieren. Im Folgenden geht es darum einen Zugang zu einer weltumspannenden, postkolonialen Perspektive zu eröffnen, die es ermöglicht die Anliegen des Globalen Südens einzubeziehen. Dies erfolgt mit Blick auf biologische, geologische und 
atmosphärische Prozesse des Planeten Erde aus einer postkolonialen, weltumspannenden Perspektive. Anders formuliert: Im Folgenden wird ein $\mathrm{Zu}-$ gang beschrieben, der die weitreichenden Implikationen der Post-/Digitalität für den Lebensalltag wie den Lebensraum sowohl für den Globalen Norden wie den Globalen Süden ausser Frage stellt und nach deren Spezifika sucht. So wie das Post- vor dem Digitalen kein Nach-der-Digitalität, sondern dessen Alternativlosigkeit thematisiert; so verweist das Post- vor dem Kolonialismus auch nicht auf ein Ende des Kolonialismus, sondern auf dessen Wiederkehr in neuer Form (im neokolonialistischen Sinne) bzw. auf die Fortsetzung historischer Abhängigkeiten in Gegenwart und Zukunft.

\section{Ein Graben nach der Post-/Digitalität aus postkolonialer Perspektive}

Die Überlegungen dieses Kapitels beginnen mit einer Enttäuschung: Der Versuch eine postkoloniale, weltumspannende Perspektive in der Medienpädagogik einzunehmen ist gewissermassen anmassend und sitzt immer einer Denktradition des Globalen Nordens auf; egal wie sehr man sich anstrengt. Hegemoniale Abhängigkeiten unterschiedlicher Gesellschaftsschichten und Weltregionen haben sich tief in unser Verständnis eingeschrieben. - Der folgende Abschnitt eröffnet einen möglichen Weg die Digitalität respektive Post-Digitalität aus postkolonialer Perspektive zu betrachten, die den Blick bewusst auf Gemeinsamkeiten und Unterschiede des Globalen Nordens und Südens in der Post-Digitalität legt.

Häufig wird im Kontext der Digitalität der Globalisierungsbegriff (bspw. in der Formel der digitalen globalen Vernetzung) bemüht, der für dieses Anliegen jedoch nicht hilfreich ist. Der Begriff bezieht sich auf die Zunahme eines Handels-, Kommunikations-, Daten- und Personenverkehrs über nationalstaatliche Grenzen hinweg (vgl. bpsw. Brodicky 2012, 2). Das vermeintliche «Zusammenschrumpfen» der Welt (Brodicky 2016, 4) oder - medienwissenschaftlich gewendet - das «Global Village» (McLuhan und Powers 1992) kann aber nur für den Globalen Norden beobachtet werden. Andernorts fehlt es an Infrastrukturen und Ausstattungen. Für die Medienpädagogik bedeutet dies, dass sich die Anliegen des technologisch gut ausgestatteten Globalen Nordens und des Globalen Südens mit 
mangelnder Infrastruktur aber gleichzeitig mit problematischen Arbeitsbedingungen etwa bei der Technologieherstellung, stark unterscheiden.

Zur kritischen Reflexion der Verhaftung in einer Denktradition des Globalen Nordens gilt es aus einer postkolonialen Perspektive weltumspannende Machtverhältnisse mitzudenken: Kolonialverhältnisse haben sich in Beziehungsverhältnisse auf Zeit eingeschrieben und sind Nährboden für neokoloniale und -imperialistische Entwicklungen (vgl. bspw. Castro Varela und Dhawan 2005). Teil des medienpädagogischen Diskurses um Post-/Digitalität sollte es daher auch sein die Re-/Produktion epistemischer Gewalt (ebd., 8) aufzuzeigen, die durch die Industrie von Informations- und Kommunikationstechnologien erwachsen ist.

Historisch gesehen haben Medien und allen voran die Schriftkultur und der Buchdruck die Kolonialisierung durch die Möglichkeit des - sprichwörtlichen - Festschreibens von Nationalstaatlichkeit vorangetrieben (ebd. 2005, 18). Auch gegenwärtig spielt Social Media eine zentrale Rolle im Kontext sozialer Teilhabe sowie bei politischen respektive demokratischen Prozessen (bspw. Wahlen). Postkolonialismus zeigt sich aber auch darin, dass sich die historische "Gier nach Rohstoffen - insbesondere Edelmetallen», die Kolonialisierung, Arbeitssklaverei und den Genozid hervorgebracht hat (Brodicky 2012, 1lf), heute in alten und neuen Abhängigkeitsverhältnissen fortschreibt. Der Bedarf an Rohstoffen und Edelmetallen, an billigen Arbeitskräften in Regionen mit niedrigem Arbeitnehmerschutz und niederschwelligen Natur- und GesundheitsschutzRegelungen zur Befriedung des Mediengebrauchs des Globalen Nordens, ist heute Motor neokolonialistischer Entwicklungen. Der Kolonialismus findet als Neokolonialismus und Rekolonialismus «immer neue Wege [...], um sich die Ressourcen der anderen Länder zu sichern» (Castro Varela und Dhawan 2005, 24).

Um nun also die Post-/Digitalität einschliesslich ihrer Implikationen für den Globalen Norden und Süden zu begreifen, bedarf es eines methodologischen Zugangs, der erlaubt die Vielfältigkeit der Digitalität zu betrachten. Nach Hepp (2018a) sei eine «materialistische Phänomenologie» hierfür zielführend. Auch ein medienarchäologischer Zugang scheint vielversprechend, der metaphorisch gesprochen, wie das Herausschürfen und Freilegen der Medienentwicklung gedacht wird. Dabei werden Spuren der Medienentwicklung bis in die Jetztzeit freigelegt. 
«Media archaeology sees media cultures as sedimented and layered, a fold of time and materiality where the past might be suddenly discovered anew, and the new technologies grow obsolete increasingly fast.» (Parikka 2012, 3)

Die Medienarchäologie ist interdisziplinär und orientiert sich an $\mathrm{Me}-$ dientheorien, den Cultural Studies, der Mediensoziologie und Medienanthropologie (Ernst 2004, 28).

Für das vorliegende Anliegen scheint insbesondere deren Auseinandersetzung mit dem Zeitalter des Anthropozäns spannend (vgl. bspw. in Parikka 2015). Im Anthropozän ist der Mensch zum grössten Einflussfaktor der biologischen, geologischen und biodynamischen Entwicklung des Planeten Erde geworden. Das Anthropozän ist das Ergebnis des menschlichen Fortschrittsstrebens und des Paradigmas des ökonomischen Wachstums (Thomay 2020, 46). Doch scheint die Diskussion über den geophysikalischen Einfluss des Menschen nicht neu. Sowohl die Beeinflussung zentraler Prozesse des Planeten Erde, als auch die Diskussion darüber beginnt bereits 1800 mit dem Beginn einer strukturellen Agrargesellschaft (Stengel 2011, 36). Zudem, so die kritischen Stimmen, würde die Idee eines Anthropozäns die Rolle des Menschen auf Erden masslos überschätzen (Bajohr 2020; Chernilo 2020). Ähnliches gilt dann wohl auch für die Idee eines "Mediazäns», in dem wiederum das «Mediale», ein alles transformierende Potential impliziert (Parikka 2018).

Doch, wie bereits gezeigt, schliesst das Konzept der Digitalität und Post-Digitalität die Veränderung des Lebensraumes auch in materieller Hinsicht ein. Entwicklung und Verbreitung digitaler Technologie verändern den Planeten Erde (vgl. Parikka 2015). Gleichermassen ermöglicht digitale Technologie aber auch ein Sichtbarmachen oder ein Bild-Machen bestimmter geophysikalischer Prozesse. So wird der Klimawandel erst durch hyper-reale Berechnungen sichtbar und die Veränderungen über grössere Zeiträume hinweg skalierbar (vgl. bspw. Chun 2015). Will man der Digitalität in einer weltumspannenden Perspektive gewahr werden, sind die «multi-layered construction[s]» der medialen Entwicklungsgeschichte freizulegen, detailliert zu beschreiben und eine «alternative» Geschichtsschreibung zu verfolgen (Parikka 2012, 1lff). Durch «zyklisches» Herausschürfen 
(vgl. Parikka 2012, 11) der Details dieser Momente, wird das jeweilige Phänomen möglichst genau beschrieben, strukturiert, neu geordnet und somit neu gedacht. Die medienarchäologische Perspektive funktioniert wie ein «Scanner», ein «Bild-in-Daten-Umwandler» (Ernst 2004, 34) und erlaubt «non-diskursive Kurzschlüsse» etwa zwischen «Technik und Gesellschaft» zu verbinden und diskursfähig zu machen (Ernst 2004, 28).

\section{Spuren eines Smart-Mobile-Device}

Der im Vorangegangen skizzierte Zugang einer postkolonialen, weltumspannenden und medienarchäologischen Hebung der Spuren der Post-/ Digitalität soll hier an Hand eines Beispiels gezeigt werden. Es handelt sich dabei um Implikationen der ubiquitären digitalen Mediennutzung und zum Zweck der Eingrenzung im Speziellen der ökologischen Nachhaltigkeit. Der Nachhaltigkeitsbegriff konzentriert sich auf ökologische, ökonomische und soziale Implikationen. Dabei geht es im Kern darum, den Planeten Erde nicht zu sehr zu zerstören respektive die Lebensqualität für nachkommende Generationen zu sichern (Harper 2001). Der Ökologiebegriff bezieht sich auf die «Beziehung der Organismen untereinander und mit ihrer Umwelt» (Schaefer 2012, 198). Ökologische Nachhaltigkeit wird also gemeinhin als Umwelt- und Klimaschutz verstanden und unterliegt zyklischen Moden und wiederkehrendem Engagement über die Zeit (vgl. Blühdorn u. a. 2020). Das Zusammendenken der Post-/Digitalität und ihrer Bedeutung für die Umwelt verlangt eine weitreichende Perspektive, die eine Vielzahl von Strängen zusammenzuführen sucht. Zur weiteren Eingrenzung wird hier als zu beschreibendes Feld ein prototypischer Lebenszyklus eines Smart-Mobile-Device fokussiert. Das Folgende zeigt die Vielzahl relevanter Aspekte im Kontext ökologischer Implikationen der Digitalität am Beispiel des Lebenszyklus eines Smart-Mobile-Device auf und bleibt aus Platzgründen kursorisch.

Die 〈Geburtsstunde〉 eines Smart-Mobile-Device liegt wohl im Zusammenspiel der Soft- und Hardware-Entwicklung, orientiert an den Bedarfen der Nutzerinnen und Nutzer und kann geographisch - etwas plakativ in Silicon Valley verortet werden: Silicon Valley als Knotenpunkt der Digitalisierungsindustrie, als Metapher des Zusammenfindens der IT-Elite 
an einem Ort. 〈Silicon〉 verweist auf Silicium und das einerseits auf den grossen Marktanteil der dort ansässigen Unternehmen in der IT- und High-Tech-Industrie und andererseits auf ökologische Auswirkungen der Ansiedelung der IT-Unternehmen in dieser Region: Dort wurden in den 1980er Jahre Chemie-Tanks deponiert, von denen giftige Chemikalien in den Boden gesickert sind. Auch in architektonischer Hinsicht manifestiert sich die Digitalität in Silicon Valley etwa am Apple Campus 2, am Google Campus und am Hauptgebäude Nvidia (vgl. u. a. Walter 2015; Donnelly 2016). Die Architektur der Gebäude, wie auch die Innenarchitektur ist an der Nutzung digitaler Medien und Infrastrukturen ausgerichtet. Aus anthropologischer Perspektive ist Silicon Valley von Interesse, wenn es um die Optimierungen respektive Manipulationen des menschlichen Körpers zur Leistungssteigerung geht. Hierfür werden chemische Substanzen konsumiert. LSD sei so nicht nur der zentrale Motor von Silicon Valley, sondern umgekehrt: Silicon Valley ein «Versuchslabor für Drogenexperimente» (Schuler 2018).

Im nächsten Schritt des Lebenszyklus eines Smart-Mobile-Device gräbt sich die Post-/Digitalisierung sprichwörtlich in tiefergehende Erdschichten ein und hinterlässt irreduzible Spuren. Dies zeigt sich vornehmlich im Globalen Süden mit hohem Edelmetall-Vorkommen: Hier treten Aspekte um Abbaubedingungen der, für die Technologieherstellung nötigen Mineralien wie insbesondere Coltan und Lithium in Erscheinung (Parikka 2015). Damit verknüpft sind Beobachtungen von Missständen der Arbeitsbedingungen, von Kinderarbeit und Gesundheitsgefährdungen durch geringe Sicherheitsstandards insbesondere bei der Verwendung chemischer Lösungsmittel. Prototypisch dafür steht die chinesische Stadt Shenzhenbai, das von den grossen Produktionshallen des Apple-Technologie-Hersteller Foxconn geprägt ist. Hier zeigen sich ebenso Hinweise auf problematische Arbeitsbedingungen und Bemühungen den Arbeitsnehmer- sowie Umweltschutz teilweise zu umgehen.

Im Globalen Süden verändert die Digitalität mit der Verwendung von Chemikalien und dem Abgraben von Erdreich den Erdboden nachhaltig. Im Globalen Norden geht es um den Technikkonsum, der durch die geplante Obsoleszenz der Geräte, durch deren sukzessives Unbrauchbarwerden bspw. durch Software-Updates und durch den Status eines Smartphones als Prestigeobjekt angefeuert wird (Remy und Huang 2014). 
Die Nutzung von Suchmaschinen und das Speichern von Daten bei Cloud-Diensten haben ebenso ökologische Auswirkungen. Die Netzkünstlerin Joana Moll hat ausgerechnet, dass die Nutzung von einer Sekunde der Suchmaschine Google 23 Bäume notwendig macht, um die CO2-Emmission wieder zu neutralisieren (Haase und Deutschlandfunk Nova 2018). ${ }^{3}$ Anders formuliert: «Mit zweihundert Suchanfragen kann man ein einziges Hemd bügeln» (Eckert 2018). Ähnlich anschauliche Beispiele finden sich über den Energieverbrauch der Datenspeicherung über Cloud-Dienste. Durch die Verbreitung des «Internets der Dinge» wird es denkbar, dass noch weitere Gegenstände «digital» werden und «online» gehen.

Der Lebenszyklus des Smart-Mobile-Device neigt sich durch seine eingeschränkte Funktionalität dem Ende zu. Häufiger jedoch werden im Globalen Norden Smartphones bereits vor Ende der Funktionstüchtigkeit ersetzt; sei es etwa aus Prestige-Gründen und/oder durch günstige Angebote von Netzbetreibern. Am ökologisch nachhaltigsten wäre es ein Gerät möglichst lange zu nutzen. Geht dies nicht mehr, gibt es Konzepte zur weiteren Verwendung (vgl. Remy und Huang 2014): So gilt es die Transfermöglichkeiten («Transferability») von einer Person zur anderen zu fördern. Hinderlich dabei sind Sperrmechanismen durch Netzbetreiber, die Sorge um private Daten auf Altgeräten und der fehlende Service durch Technologiehersteller bei der Weiterverwendung. Zur längerfristigen Nutzung von Smart-Mobile-Devices seien diese grundsätzlich so zu bauen, dass sie von Laien, ggf. mit einfachen Anleitungen und mit handelsüblichen Werkzeugen prinzipiell repariert werden könnten. Zudem gibt es unter dem Schlagwort der «Augmentation» Überlegungen Altgeräte mit leichter Modifikation in anderer Funktion weiter zu nutzen. Und schliesslich können die Materialien («Re-use of Materials») weiterverwendet werden, indem sie lokalen Elektroschott-Entsorgungsnetzwerken bzw. Wiederaufbereitungs-Unternehmen zugänglich gemacht werden. Doch bei alle dem: Studien haben gezeigt, dass Altgeräte eher ungenutzt ablegt, als dass diese einer Weiternutzung oder -verwendung der Materialien zugeführt werden (Remy und Huang 2014, 6).

3 Dies veranschaulicht die Visualisierung «DEFOOO০০০০০০০০০০০০০০০OO-OREST» http://www.janavirgin.com/CO2/DEFO00000000000000000OOOREST.html der Künstlerin Moll (abgerufen am 28.04.2019). 
Die Post-/Digitalität verändert unseren Lebensraum und damit auch den Planeten Erde für nachkommende Generationen nachhaltig. Sie gräbt sie sich - metaphorisch gesprochen - in die Erde ein (Parikka 2015), verändert die Erdoberfläche, die Architektur, das Klima und den Menschen.

\section{Conclusio: Anschlussstellen für die Medienpädagogik}

Aktuell nehmen Forschungsvorhaben, Publikationen, Empfehlungen und Veranstaltungen zu ökologischen Implikationen der 〈Digitalisierung〉 oder Post-/Digitalität zu. So könnte man schon von einem Trend sprechen, der mit Blühdorn (2020) durchaus als Neuauflage des immer wiederkehrenden Diskurses um Umwelt- und Klimaschutz verstanden werden kann.

Innerhalb der Medienpädagogik als Praxisfeld zeigen sich vermehrt Initiativen, etwa in der Entwicklung und Bereitstellung von Lehr-Lernmaterialien ${ }^{4}$. Im Zentrum steht dabei die Ermöglichung eines Bewusstseins über die ökologische Dimension der Nutzung digitaler Medien sowie das Übersetzen dessen in das subjektive Medienhandeln. Auch auf politischprogrammatischer Ebene finden sich die Anliegen verschriftlicht ${ }^{5}$, wobei

4 Siehe dazu exemplarisch das Projekt ÖHA! - Ökologisch-nachhaltiges Medienhandeln in Schule und darüber hinaus der Pädagogischen Hochschule Wien (https://oeha.phwien.ac.at/) oder die Materialien Digitalisierung und Klima von Saferinternet.at (www.saferinternet.at/fileadmin/categorized/Materialien/Digitalisierung_und_Klima.pdf)

5 Auf EU-Ebene wird im DigComp-Katalog auf Ebene 4.4 "Protecting the environment. To be aware of the environmental impact of digital technologies and their use.» angeführt (Carretero, Vuorikari, und Punie 2017, 17). Für den schulischen Kontext in Deutschland findet sich der Punkt «4.4. Natur und Umwelt schützen» (genauer: «4.4.1. Umweltauswirkungen digitaler Technologien berücksichtigen Problemlösen und Handeln») in der KMK-Strategie "Bildung in der digitalen Welt» (KMK 2016). Für Österreich findet sich im Lehrplan der verbindlichen Übung «Digitale Grundbildung» in der Sekundarstufe unter «Gesellschaftliche[n] Aspekte von Medienwandel und Digitalisierung» einerseits das Ziel, dass Schüler*innen «die Dynamik und Bedeutung von Werten, Normen und unterschiedlichen Interessen im Hinblick auf die Nutzung von digitalen Medien (ökonomisch, religiös, politisch, kulturell) [kennen]» sowie darüber Bescheid «wissen, inwieweit die Nutzung digitaler Technologien der Umwelt schadet oder zum Umweltschutz beiträgt» (Bundesgesetzblatt für die Republik Österreich 2018). 
dies bekanntermassen nicht immer zu einer tatsächlich stärkeren Thematisierung im medien-/pädagogischen Praxisfeld führt.

Im medienpädagogischen Kontext finden sich dazu bis dato wenige wissenschaftliche Arbeiten. Zwar gibt es eine längere Tradition der Auseinandersetzung mit dem Verhältnis von Mensch, Natur und Technik respektive Medien (vgl. bspw. Meyer-Drawe 1996; Euler 2009). Ebenso erfährt die Disziplin «Bildung für nachhaltige Entwicklung» (vgl. bspw. Grundmann 2017; Stoltenberg und Burandt 2014) sowie der Kontext «Globales Lernen» (vgl. bspw. Brendel, Schrufer, und Schwarz 2018) gegenwärtig einen Aufschwung und beginnt sich auch mit der Digitalisierung respektive PostDigitalität auseinander zusetzen. In der Medienpädagogik als wissenschaftlichen Disziplin zeigt sich bis dato aber noch keine systematische Auseinandersetzung. Mit Blick auf post- und neokoloniale Entwicklungen innerhalb der Post-/Digitalität und der Frage ökologischer, ökonomischer und sozialer Implikationen dieser, eröffnet sich ein zentrales Forschungsfeld angesichts der humanitären und ökologischen Herausforderungen der Gegenwart. Dieses Forschungsfeld legt interdisziplinäre Zugänge (vgl. bspw. Castro Varela und Dhawan 2005, 8, 12, 24; Hepp 2014) und damit eine Öffnung der Medienpädagogik bspw. gegenüber der Medienwissenschaft, aber auch gegenüber «Bildung für nachhaltiger Entwicklung» und den Postcolonial Studies nahe.

Im Zentrum steht die Ermöglichung eines Bewusstseins über Implikationen der Digitalität respektive Post-Digitalität für den Lebensalltag und Lebensraum. Dabei konsequent die reine Perspektive des Globalen Nordens zu verlassen, scheint ein spannendes und aktuell hoch relevantes Forschungsfeld. Dies aus zwei Gründen: Einerseits sind aktuell die meisten wertvollen Rohstoffe wie Edelmetalle in Geräten verbaut, die noch genutzt werden (Thiébaud u. a. 2018, 12). Erfolgt jetzt eine Sensibilisierung zu einem ökologisch-nachhaltigeren Medienhandeln, ergibt sich theoretisch die Chance diese Rohstoffe einer Wiederaufbereitung und Weiterverwendung zuzuführen. Und andererseits verweisen aktuelle technologische Entwicklungen - Stichwort: smart everything - darauf, dass in Zukunft noch mehr digitale Geräte pro Kopf im Umlauf sein werden, die ihrerseits dann wiederum weitere ökologische, ökonomische und soziale Herausforderungen mit sich bringen. 


\section{Literatur}

Bajohr, Hannes. 2020. Der Anthropos im Anthropozän: Die Wiederkehr des Menschen im Moment seiner vermeintlich endgültigen Verabschiedung. Berlin; Boston: De Gruyter,. https://doi.org/10.1515/9783110668551.

Bauman, Zygmunt. 2000. Flüchtige Moderne. Übersetzt von Reinhard Kreissl. Frankfurt am Main: Suhrkamp (2003).

Blühdorn, Ingolfur, Michael Deflorian, Mirijam Mock, Daniel Hausknost, und Felix Butzlaff. 2020. Nachhaltige Nicht-Nachhaltigkeit: Warum die ökologische Transformation der Gesellschaft nicht stattfindet. X-Texte zu Kultur und Gesellschaft. transcript Verlag. https://doi.org/10.14361/9783839445167.

Brendel, Nina, Gabriele Schrufer, und Ingrid Schwarz. 2018. Globales Lernen im digitalen Zeitalter. Münster: Waxmann.

Brodicky, Sophie. 2012. «Pädagogik im Spannungsfeld der Globalisierung. Die süßsaure Herausforderung im ‘Zwischen〉». Herausgegeben von Heidi Grobbauer, Hakan Gürses, und Stefan Vater. Magazin Erwachsenenbildung.at Global Lernen. Zugänge (16): 4/1-8.

Bundesgesetzblatt für die Republik Österreich. 2018. «Änderung der Verordnung über die Lehrpläne der Neuen Mittelschulen sowie der Verordnung über die Lehrpläne der allgemeinbildenden höheren Schulen». https://www.ris.bka. gv.at/Dokumente/BgblAuth/BGBLA_2018_II_71/BGBLA_2018_II_71.rtf.

Bundesministerium für Bildung, Wissenschaft und Forschung. 2018. «Masterplan Digitalisierung». Wien. https://bmbwf.gv.at/fileadmin/user_upload/Aussendung/Masterplan_Digitalisierung/Masterplan_Digitalisierung_Presseinformation.pdf.

Carretero, Stephanie, Riina Vuorikari, und Yves Punie. 2017. DigComp 2.1. The Digital Competence Framework for Citizens with eight proficiency levels and examples of use. Publications Office of the European Union. Luxembourg. http://publications.jrc.ec.europa.eu/repository/bitstream/JRC106281/webdigcomp2.lpdf_(online).pdf.

Castro Varela, María do Mar, und Nikita Dhawan, Hrsg. 2005. Postkoloniale Theorie - Eine kritische Einführung. Bielefeld: transcript. https://doi. org/10.14361/9783839411483.

Chernilo, Daniel. 2020. «Die Frage nach dem Menschen in der Anthropozändebatte». In Der Anthropos im Anthropozän: Die Wiederkehr des Menschen im Moment seiner vermeintlich endgültigen Verabschiedung, herausgegeben von Hannes Bajohr, 55-76. Berlin; Boston: De Gruyter. https://doi.org/10.1515/9783110668551.

Chun, Wendy Hui Kyong. 2015. «On Hypo-Real Models or Global Climate Change: A Challenge for the Humanities». Critical Inquiry, The University of Chicago Press, 41 (3): 675-703. https://www.jstor.org/stable/10.1086/680090.

Cramer, Florian. 2014. «What Is 'Post-Digital'?» APRJA 3 (1):11-24. https://doi. org/10.7146/aprja.v3i1.116068. 
Cramer, Florian. 2016. «Nach dem Koitus oder nach dem Tod? Zur Begriffsverwirrung von "Post-digital", "Post-Internet" und „Post-Media"». Kunstforum International 242 (Oktober): 54-67. https://www.kunstforum.de/artikel/nachdem-koitus-oder-nach-dem-tod/.

Deutsche Kultusministerkonferenz. 2016. «Bildung in der digitalen Welt. Strategie der Kultusministerkonferenz». Berlin: Sekretariat der Kultusministerkonferenz. Www.kmk.org/fileadmin/Dateien/pdf/PresseUndAktuelles/2016/ Bildung_digitale_Welt_Webversion.pdf.

Donnelly, Erin. 2016. «The Silicon Valley Architecture Boom: 3 New HQs». Azure Magazine. 22. September 2016. https://www.azuremagazine.com/article/silicon-valley-architecture-boom/.

Eckert, Werner. 2018. «Faktencheck: Ökobilanz von Suchmaschinen | SWR Wissen». swr.online. 4. September 2018. https://www.swr.de/wissen/20jahre-google-umweltfacts-zu-suchmaschinen/-/id=253126/did=22378814/ nid=253126/d2azhl/index.html.

Ernst, Wolfgang. 2004. «Der medienarchäologische Blick». In Die Medien und ihre Technik: Theorien, Modelle, Geschichte, herausgegeben von Harro Segeberg, 28-42. Schriftenreihe der Gesellschaft für Medienwissenschaft (GFM); 11. Marburg: Schüren.

Euler, Peter. 2009. "Bildung und Nachhaltigkeit - Historische Ursprünge und ökonomische Hintergründe einer widerspruchsreichen Beziehung». Beiträge Region und Nachhaltigkeit. Zu Forschung und Entwicklung im UNESCO-Biosphärenreservat Rhön; 6. Jahrgang, Heft 6/2009; Fulda. 103-111. http://nbn-resolving.de/urn:nbn:de:hebis:66-opus-1420.

Faßler, Manfred. 2009. Nach der Gesellschaft: infogene Welten, anthropologische Zukünfte. München: Wilhelm Fink.

Faßler, Manfred. 2011. Kampf der Habitate: Neuerfindungen des Lebens im 21. Jahrhundert. Wien und New York: Springer.

Faßler, Manfred. 2014. Das Soziale. Entstehung und Zukunft menschlicher Selbstorganisation. Paderborn: Wilhelm Fink.

Grünberger, Nina. 2020. «Klimaschutz und Digitalisierung als medienpädagogische Verantwortung?» In Bildung und Digitalisierung: Auf der Suche nach Kompetenzen und Performanzen, herausgegeben von Christine Trültzsch-Wijnen und Gerhard Brandhofer, 1. Aufl., 181-94. Baden-Baden: Nomos. https://doi. org/10.5771/9783748906247-181.

Grundmann, Diana. 2017. Bildung für nachhaltige Entwicklung in Schulen verankern. Wiesbaden: Springer VS.

Haase, Till, und Deutschlandfunk Nova. 2018. «23 Bäume, um eine Sekunde googeln wiedergutzumachen». Deutschlandfunk Nova. 9. Mai 2018. https://www. deutschlandfunknova.de/beitrag/co2-abdruck-jede-sekunde-googeln-verbraucht-23-baeume.

Harper, Douglas. 2001. «Sustainable (adj.)». In Online Etymology Dictionary. https:// www.etymonline.com/word/sustainable. 
Hepp, Andreas. 2014. «Mediatisierung / Medialisierung». In Handbuch Medienwissenschaft, von Jens Schröter. Stuttart, Weimar: Metzler Verlag.

Hepp, Andreas. 2018a. «Von der Mediatisierung zur tiefgreifenden Mediatisierung: Konstruktivistische Grundlagen und Weiterentwicklungen in der $\mathrm{Me}-$ diatisierungsforschung». In Kommunikation - Medien - Konstruktion. Braucht die Mediatisierungsforschung den Kommunikativen Konstruktivismus?, von Jo Reichertz und Richard Bettmann, 27-45. Wiesbaden: Springer VS.

Hepp, Andreas. 2018b. «Die kommunikative Konstruktion der Wirklichkeit - oder: Sozial- und Gesellschaftstheorie in Zeiten tiefgreifender Mediatisierung». Soziologische Revue. Besprechungen neuer Literatur 41 (2): 198-207.

Jörissen, Benjamin, und Winfried Marotzki. 2009. Medienbildung - Eine Einführung. 1. Aufl. Bad Heilbrunn: UTB.

Lyotard, Jean-François. 1979. Das postmoderne Wissen: Ein Bericht. Übersetzt von Otto Pfersmann. 7., ü.a. Aufl. Wien: Passagen Verlag (2012).

McLuhan, Marshall, und Bruce R. Powers. 1992. The Global Village: Transformations in World Life and Media in the 21st Century. Oxf Univ PR Pbk. New York: Oxford University Press, U.S.A.

Meder, Norbert. 2004. Der Sprachspieler: Der postmoderne Mensch oder das Bildungsideal im Zeitalter der neuen Technologien. 2. wesentl. erw. Aufl. Würzburg: Königshausen und Neumann.

Meyer-Drawe, Käte. 1996. Menschen im Spiegel ihrer Maschinen. München: Fink.

Murray, Sarah. 2020. «Postdigital Cultural Studies». International Journal of Cultural Studies 23 (4): 441-50. https://doi.org/10.1177/1367877920918599.

Parikka, Jussi. 2012. What is Media Archaeology? Cambridge: Polity Press.

Parikka, Jussi. 2015. A Geology of Media. Minneapolis; London: Electronic Mediations.

Parikka, Jussi. 2018. «Medianatures》. In FOCUS Medioscene, herausgegeben von Lorenz Engell und Bernhard Siegert, 9/1 (2018):103-6. Zeitschrift für Medienund Kulturforschung. Hamburg: Felix Meiner Verlag.

Remy, Christian, und Elaine May Huang. 2014. "Addressing the obsolescence of end-user devices: Ap- proaches from the field of sustainable HCI». In ICT Innovations for Sustainability, von Lorenz M. Hilty und Bernhard Aebischer, 257-67. Heidelberg/New York: Springer. https://doi.org/10.1007/978-3-319-09228-7_15.

Schaefer, Matthias. 2012. Wörterbuch der Ökologie. 5. neu bearbeitete und erweiterte Auflage. Heidelberg: Spektrum Akademischer Verlag. https://doi. org/10.1007/978-3-8274-2562-1.

Schuler, Marcus. 2018. «Drogen und Essstörungen im Silicon Valley - Der Preis der Selbstoptimierung». Deutschlandfunk Kultur. 2. Juli 2018. https://www. deutschlandfunkkultur.de/drogen-und-essstoerungen-im-silicon-valley-derpreis-der.979.de.html?dram:article_id=421328.

Stalder, Felix. 2019. Kultur der Digitalität. 4. Aufl. 2019. Berlin: Suhrkamp.

Stengel, Oliver. 2011. Suffizienz. Die Konsumgesellschaft in der ökologischen Krise. München: oekom Verlag. 
Stoltenberg, Ute, und Simon Burandt. 2014. «Bildung für eine nachhaltige Entwicklung». In Nachhaltigkeitswissenschaften, herausgegeben von Harald Heinrichs und Gerd Michelsen, 567-94. Berlin, Heidelberg: Springer. https://doi. org/10.1007/978-3-642-25112-2_17.

Swertz, Christian, Wolfgang Benjamin Ruge, Alexander Schmölz, und Alessandro Barberi, Hrsg. 2017. Die Konstitution der Medienpädagogik. Zwischen interdisziplinärem Forschungsfeld und bildungswissenschaftlicher (Sub-) Disziplin. Medien Pädagogik. Zeitschrift für Theorie und Praxis der Medienpädagogik. 29. https://doi.org/10.21240/mpaed/29.X.

Thiébaud, Esther, Lorenz M. Hilty, Mathias Schluep, Heinz W. Böni, und Martin Faulstich. 2018. «Where Do Our Resources Go? Indium, Neodymium, and Gold Flows Connected to the Use of Electronic Equipment in Switzerland». Sustainability 10/8. https://doi.org/10.3390/su10082658.

Thomay, Marius. 2020. «Degrowth und der (Eigen-)Wert der Natur: Eine kritische Reflexion umweltethischer Positionen in der Degrowth-Bewegung und der Versuch eines Plädoyers für eine holistische Umweltethik». In Nachhaltigkeit, Postwachstum, Transformation, herausgegeben von Ulrich Roos, 45-82. Wiesbaden: Springer Fachmedien Wiesbaden. https://doi.org/10.1007/978-3-65829973-6_3.

Trültzsch-Wijnen, Christine, Hrsg. 2017. Medienpädagogik. Eine Standortbestimmung. Bd. 1. Medienpädagogik | Media Education. Baden-Baden: Nomos.

Walter, Alexander. 2015. «Apple's next, HOK-Designed Silicon Valley Spaceship Revealed». Archinect News, 2. Oktober 2015. https://archinect.com/news/article/138032380/apple-s-next-hok-designed-silicon-valley-spaceship-revealed. 